\title{
Impact of sarcopenia on long-term mortality and cardiovascular events in patients undergoing hemodialysis
}

Jwa-Kyung Kim ${ }^{1}$, Sung Gyun Kim ${ }^{1}$ Ji-Eun $\mathrm{Oh}^{2}$, Young-Ki Lee ${ }^{3}$, Jung-Woo Noh³ ${ }^{3}$ Hyung Jik Kim ${ }^{1}$, and Young Rim Song ${ }^{1}$

\author{
'Department of Internal Medicine, \\ Hallym University Sacred Heart \\ Hospital, Anyang; ${ }^{2}$ Department \\ of Internal Medicine, Hallym \\ University Kangdong Sacred Heart \\ Hospital, Seoul; ${ }^{3}$ Department of \\ Internal Medicine, Hallym University \\ Kangnam Sacred Heart Hospital, \\ Seoul, Korea
}

Background/Aims: A high body mass index (BMI) is known to correlate with better survival in patients on hemodialysis (HD). However, the impacts of body composition and sarcopenia on survival have not been well studied in this population.

Methods: One hundred and forty-two prevalent HD patients were recruited and followed prospectively for up to 4.5 years. Low muscle mass (measured using a portable, whole-body, bioimpedance spectroscopic device) was defined as a lean tissue index (LTI) two standard deviations (SD) or more below the normal gender-specific mean for young people. Low muscle strength was a handgrip strength (HGS) of less than $30 \mathrm{~kg}$ in males and less than $20 \mathrm{~kg}$ in females. Sarcopenia was considered present when both LTI and HGS were reduced.

Results: The mean age was $59.8 \pm 13.1$ years; $57.0 \%$ were male and $47.2 \%$ had diabetes. Forty-seven patients (33.1\%) had sarcopenia. During follow-up, 28 patients (19.7\%) died, and low LTI (adjusted hazard ratio [HR], 2.77; 95\% confidence interval [CI], 1.10 to 6.97) and low HGS (HR 5.65; 95\% CI, 1.99 to 16.04) were independently associated with mortality. Sarcopenia was a significant predictor for death (HR, 6.99; $95 \% \mathrm{CI}, 1.84$ to $26.58 ; p=0.004$ ) and cardiovascular events (HR, $4.33 ; 95 \% \mathrm{CI}$, 1.51 to $12.43 ; p=0.006)$.

Conclusions: Sarcopenia was strongly associated with long-term mortality and cardiovascular events in HD patients. Assessment of muscle strength and muscle mass may provide additional prognostic information to survival in patients with end-stage renal disease.

Keywords: Cardiovascular diseases; Kidney failure, chronic; Hand strength; Sarcopenia; Mortality

\section{INTRODUCTION}

Protein-energy wasting (PEW) is a common complication in patients with chronic kidney disease (CKD), and is considered to be an important predictor of low quality of life, poor cardiovascular outcomes, and mortality [1].
Skeletal muscle protein accounts for $50 \%$ to $60 \%$ of all body protein and is involved in many metabolic functions. Therefore, muscle mass and strength have been suggested to be useful markers of both nutritional status and PEW.

Sarcopenia is defined by progressive declines in mus- 
cle mass and strength upon aging [2], and is also apparent in patients (even younger ones) with chronic disease [3]. Loss of muscle mass and strength commences in the fourth decade of life and the declines approach 50\% in the eighth decade [2]. Patients with chronic disease probably experience similar age-associated changes in bodily composition, but muscle mass loss in such patients is much more marked and occurs earlier than in subjects who do not have chronic illnesses. A high body mass index (BMI) is associated with better outcomes in patients with end-stage renal disease (ESRD) [4-8]. However, the BMI does not differentiate lean from fat mass and is less correlated with muscle mass in ESRD patients than in others [9]. Reductions in both muscle mass and strength are common and significantly affect both the morbidity and the mortality of dialysis patients [10-12]. In our previous study, we found that BMI was associated with fat mass rather than muscle mass; we also showed that muscle loss with relative conservation of fat mass is common in patients with CKD [13]. Although a high BMI has consistently been shown to correlate with better survival, the impacts of body composition and sarcopenia on survival have not been well studied in ESRD patients. It has been suggested that muscle strength may be more important than muscle mass when evaluating sarcopenia; low muscle strength was more strongly associated with mortality than was low muscle mass [14]. Physical performance and muscle strength were significant risk factors for mortality in patients with ESRD [12,15]. Despite growing recognition of the importance of muscle quality, only a few studies have evaluated the association between muscle mass or strength and survival in dialysis patients. We previously reported that sarcopenia was prevalent in elderly patients with ESRD, and was associated with inflammation, depression, and cognitive dysfunction [13]. In the present study, we defined sarcopenia by muscle mass and handgrip strength (HGS) and evaluated the impact of sarcopenia on the mortality and cardiovascular events of adult patients on maintenance hemodialysis (HD).

\section{METHODS}

\section{Study subjects}

This was a prospective observational study on 142 pa- tients undergoing HD in three dialysis units of Hallym University Sacred Heart Hospital, South Korea. The patients were recruited between January 2011 and December 2011. All patients had been on HD for at least 3 months. The exclusion criteria were: age less than 18 years; an active infection or bleeding within 3 months prior to enrolment; a history of malignancy or a chronic inflammatory disease; the presence of any implantable device including a cardiac pacemaker or defibrillator, or a metallic implant; amputee status; and pregnancy. All patients had been on regular HD three times weekly for 4 hours each time. The study was approved by the Ethics Review Committee of our hospital (IRB No: 2012-Io53) and was conducted in compliance with tenets of the Declaration of Helsinki. All patients gave written informed consent for participation in the study and data processing.

Blood samples were collected for biochemical tests immediately before a midweek HD session, and the serum levels of albumin, prealbumin, glucose, uric acid, and parathyroid hormone were measured using routine laboratory methods. High-sensitivity C-reactive protein (hs-CRP) levels were measured with the aid of a BN II analyzer (Dade Behring, Newark, DE, USA) running a latex-enhanced immunonephelometric method.

\section{Nutritional assessment}

To assess nutritional status, we measured albumin and prealbumin levels, performed Subjective Global Assessment (SGA), and collected anthropometric data (height, weight, BMI, and triceps skin-fold thickness). BMI was calculated using the following equation: BMI = weight/ height ${ }^{2}\left(\mathrm{~kg} / \mathrm{m}^{2}\right)$. Triceps skin-fold thicknesses were obtained immediately after HD sessions by a single trained clinician using a Lange skin-fold caliper (Beta Technology Inc., Cambridge, MD, USA). To measure the SGA, five components of medical history (weight change, dietary intake, gastrointestinal symptoms, functional capacity, and comorbid conditions and their relationships to nutritional requirements) and three components of a brief physical examination (signs of fat and muscle wasting, alterations in fluid balance) were evaluated using a seven-point scoring system [16].

\section{Measurements of body composition and muscle strength}

Body composition was assessed using a portable, whole- 
body, bioimpedance spectroscopic device (Body Composition Monitor, Fresenius Medical Care, Bad Homburg, Germany). The instrument yields objective indicators of muscle mass (lean tissue mass, LTM), fat mass, and hydration status. LTM and fat mass levels were normalized to the body surface area $\left(\mathrm{m}^{2}\right)$ to obtain a lean tissue index (LTI) and fat tissue index (FTI). Low muscle mass was defined as an LTI two standard deviations (SDs) or more below the normal gender-specific means for young people. To determine muscle strength, the HGS of the non-fistulated hand after a dialysis session using a Jamar handheld dynamometer (JAMAR PLUS+, Sammons Preston Inc., Bolingbrook, IL, USA). All subjects stood with both arms extended sideways from the body, with the dynamometer facing away from the body. Three trials were performed with a rest period of at least 1 minute between trials, and averages were calculated. Low muscle strength was classified as an HGS below 30 $\mathrm{kg}$ in males and below $20 \mathrm{~kg}$ in females.

\section{Statistical analysis}

All statistical analyses were performed using SPSS version 18.0 (SPSS Inc., Chicago, IL, USA). Continuous variables are expressed as means \pm SDs and categorical variables as numbers and percentages. The Kolmogorov-Smirnov test was used to explore the normality of distribution. Skewed data, including hs-CRP levels, were converted to natural logarithms. Pearson correlation analysis was used to clarify the relationships between body compositions and biochemical and nutritional parameters. Logistic regression tests were performed to explore correlations among the assessed parameters. Survival curves were plotted using the Kaplan-Meier method and evaluated with the aid of the log-rank test. The hazard ratios (HRs) for mortality were determined via both crude and multivariate Cox regression analyses and are presented with $95 \%$ confidence intervals (CIs). A p value < 0.05 was deemed to indicate statistical significance.

\section{RESULTS}

\section{Patients characteristics}

The mean patient age was $59.8 \pm 13.1$ years (range, 21 to 88 ). Eighty-one patients (57.0\%) were male and 67 (47.2\%) had diabetes. The mean time on dialysis was 4.2 \pm 4.0 years. The mean follow-up duration was $4.3 \pm 0.8$ years. Forty-seven patients (33.1\%) had sarcopenia and $68(47.9 \%)$ both an appropriate LTI and HGS. Twenty patients (14.1\%) had low muscle mass but appropriate muscle strength and 8 (5.6\%) exhibited low muscle strength but appropriate muscle mass.

Table 1 shows baseline characteristics by sarcopenic status. No significant among-group difference was evident in any of gender, age, the prevalence of diabetes, BMI, the duration of dialysis, the cause of ESRD, or a previous history of coronary artery disease (CAD) or cerebrovascular disease (CVD). The serum levels of hemoglobin, cholesterol, intact parathyroid hormone, albumin, and prealbumin were similar between the groups. However, the serum level of 25-hydroxyvitamin $\mathrm{D}$ was lower, and the serum levels of $\beta 2$-microglobulin, glycated hemoglobin ( $\mathrm{HbAlc})$, and hs-CRP were higher, in patients with than without sarcopenia. Among the nutritional parameters, only the SGA score differed between the two groups. Of the 47 patients with sarcopenia, only 12 patients were underweight $\left(\mathrm{BMI}<2 \mathrm{O} \mathrm{kg} / \mathrm{m}^{2}\right)$ and five patients were overweight $\left(\mathrm{BMI}>25 \mathrm{~kg} / \mathrm{m}^{2}\right)$.

\section{Baseline nutritional status}

Baseline nutritional parameters by survival status are shown in Table 2. No significant between-group difference was evident in any of BMI, triceps skin-fold thickness, or the FTI. However, the HGS, the LTI, and the serum prealbumin level were significantly lower in patients who died. Table 3 shows correlates between body compositional and nutritional parameters. BMI and triceps skin-fold thickness were strongly associated only with the FTI, thus not with the LTI. Conversely, the SGA was closely associated with the LTI $(r=0.243, p=0.019)$ and the HGS $(r=0.347, p=0.001)$, but not with the FTI. The serum levels of prealbumin and albumin were not associated with any of the LTI, HGS, or FTI. The serum level of hs-CRP was associated with the HGS, but not the FTI or LTI. Multivariate logistic regression analysis revealed that the LTI was significantly associated with only the SGA score, and the HGS only with the hs-CRP level and the SGA score. Among the biochemical, nutritional, and anthropometric parameters, the hs-CRP level (odds ratio [OR], 1.95; 95\% CI, 1.26 to 3.02; $p=0.003$ ), the SGA (OR, $0.45 ; 95 \% \mathrm{CI}, 0.21$ to $0.96 ; p=0.039)$, and the $\beta 2$-microglobulin level (OR, 1.11; 95\% CI, 1.01 to 1.21; $p=$ 
0.025) were significantly associated with sarcopenia after adjusting for age, sex, diabetes status, CAD history, CVD history, BMI, and the albumin and hs-CRP levels.

\section{Multivariate predictors of all-cause mortality}

During the follow-up period, 28 patients died: 10 of cardiovascular disease, 13 of infection, three of CVD, and two of malignancy. Fig. 1 shows the Kaplan-Meier surviv-

Table 1 Baseline characteristics of study subjects

\begin{tabular}{|c|c|c|c|}
\hline Variable & Sarcopenia $(\mathrm{n}=47,33.1 \%)$ & No sarcopenia $(\mathrm{n}=95,66.9 \%)$ & $p$ value \\
\hline Age, yr & $62.4 \pm 14.0$ & $59.2 \pm 13.0$ & 0.178 \\
\hline Male sex & $24(51.1)$ & $57(60.0)$ & 0.311 \\
\hline Body mass index, $\mathrm{kg} / \mathrm{m}^{2}$ & $21.9 \pm 2.8$ & $22.7 \pm 3.5$ & 0.146 \\
\hline Triceps muscle thickness, mm & $1.0 \pm 0.5$ & $1.1 \pm 0.3$ & 0.485 \\
\hline Cause of end-stage renal disease & & & 0.541 \\
\hline Diabetic & $29(61.7)$ & $48(50.5)$ & \\
\hline Hypertensive & $12(25 \cdot 5)$ & $25(26.3)$ & \\
\hline Glomerulonephritis & $3(6.4)$ & $14(14.7)$ & \\
\hline Others & $3(6.4)$ & $8(8.5)$ & \\
\hline Coronary artery disease & $14(29.8)$ & $21(22.1)$ & 0.502 \\
\hline Cerebrovascular disease & $14(29.8)$ & $19(20.0)$ & 0.326 \\
\hline Duration of dialysis, mon & $50.7 \pm 41.9$ & $50.0 \pm 50.6$ & 0.934 \\
\hline Mean arterial pressure, $\mathrm{mmHg}$ & $98.1 \pm 22.2$ & $105.0 \pm 11.4$ & 0.053 \\
\hline Hemoglobin, g/dL & $10.3 \pm 1.0$ & $10.0 \pm 0.8$ & 0.099 \\
\hline Glucose, mg/dL & $130.0 \pm 90$ & $112.7 \pm 49.2$ & 0.198 \\
\hline Albumin, g/dL & $3.7 \pm 0.3$ & $3.8 \pm 0.4$ & 0.580 \\
\hline Prealbumin, mg/dL & $23.5 \pm 10.9$ & $27 \cdot 3 \pm 8.0$ & 0.103 \\
\hline $\mathrm{HDL}-\mathrm{C}, \mathrm{mg} / \mathrm{dL}$ & $44.5 \pm 13.1$ & $44.8 \pm 11.6$ & 0.900 \\
\hline LDL-C, mg/dL & $74.8 \pm 30.5$ & $79.5 \pm 24.8$ & 0.390 \\
\hline Triglyceride, mg/dL & $92.1 \pm 55.6$ & $85.0 \pm 43.0$ & 0.465 \\
\hline Uric acid, mg/dL & $10.3 \pm 16.5$ & $7.7 \pm 1.5$ & 0.355 \\
\hline Serum ferritin, $\mu \mathrm{g} / \mathrm{L}$ & $214.4 \pm 167 \cdot 3$ & $199.8 \pm 110.4$ & 0.590 \\
\hline Intact parathyroid hormone, $\mathrm{pg} / \mathrm{mL}$ & $175.9 \pm 270.9$ & $171.7 \pm 197.9$ & 0.929 \\
\hline ß2-Microglobulin, mg/L & $29.2 \pm 9.2$ & $24.7 \pm 7.1$ & 0.013 \\
\hline 25-Hydroxyvitamin $\mathrm{D}, \mathrm{ng} / \mathrm{mL}$ & $7 \cdot 4 \pm 4.0$ & $10.4 \pm 5.0$ & 0.002 \\
\hline hs-CRP ${ }^{\mathrm{a}}$ & $0.8 \pm 1.3$ & $-0.2 \pm 1.2$ & 0.001 \\
\hline HbAıc, \% & $7 \cdot 5 \pm 1.4$ & $6.8 \pm 1.1$ & 0.038 \\
\hline $\mathrm{spKt} / \mathrm{V}$ & $1.5 \pm 0.4$ & $1.5 \pm 0.2$ & 0.668 \\
\hline nPCR, g/kg/day & $1.1 \pm 0.3$ & $1.1 \pm 0.3$ & 0.919 \\
\hline SGA, score & $5 \cdot 3 \pm 1.4$ & $6.1 \pm 0.7$ & 0.008 \\
\hline Overhydration, L & $0.8 \pm 2.4$ & $0.2 \pm 2.6$ & 0.725 \\
\hline Lean tissue index, $\mathrm{kg} / \mathrm{m}^{2}$ & $11.0 \pm 2.2$ & $13.9 \pm 2.9$ & $<0.001$ \\
\hline Fat tissue index, $\mathrm{kg} / \mathrm{m}^{2}$ & $10.6 \pm 3.7$ & $8.4 \pm 4.3$ & 0.004 \\
\hline Handgrip strength, kg & $18.2 \pm 6.5$ & $27.2 \pm 8$ & $<0.001$ \\
\hline
\end{tabular}

Values are presented as mean \pm SD or number (\%).

HDL-C, high density lipoprotein cholesterol; LDL-C, low density lipoprotein cholesterol; hs-CRP, high-sensitivity C-reactive protein; HbAlc, glycated hemoglobin; spKt/V, single pool Kt/V; nPCR, normalized protein catabolism rate; SGA, Subjective Global Assessment.

${ }^{\mathrm{a}}$ Log-transformed. 

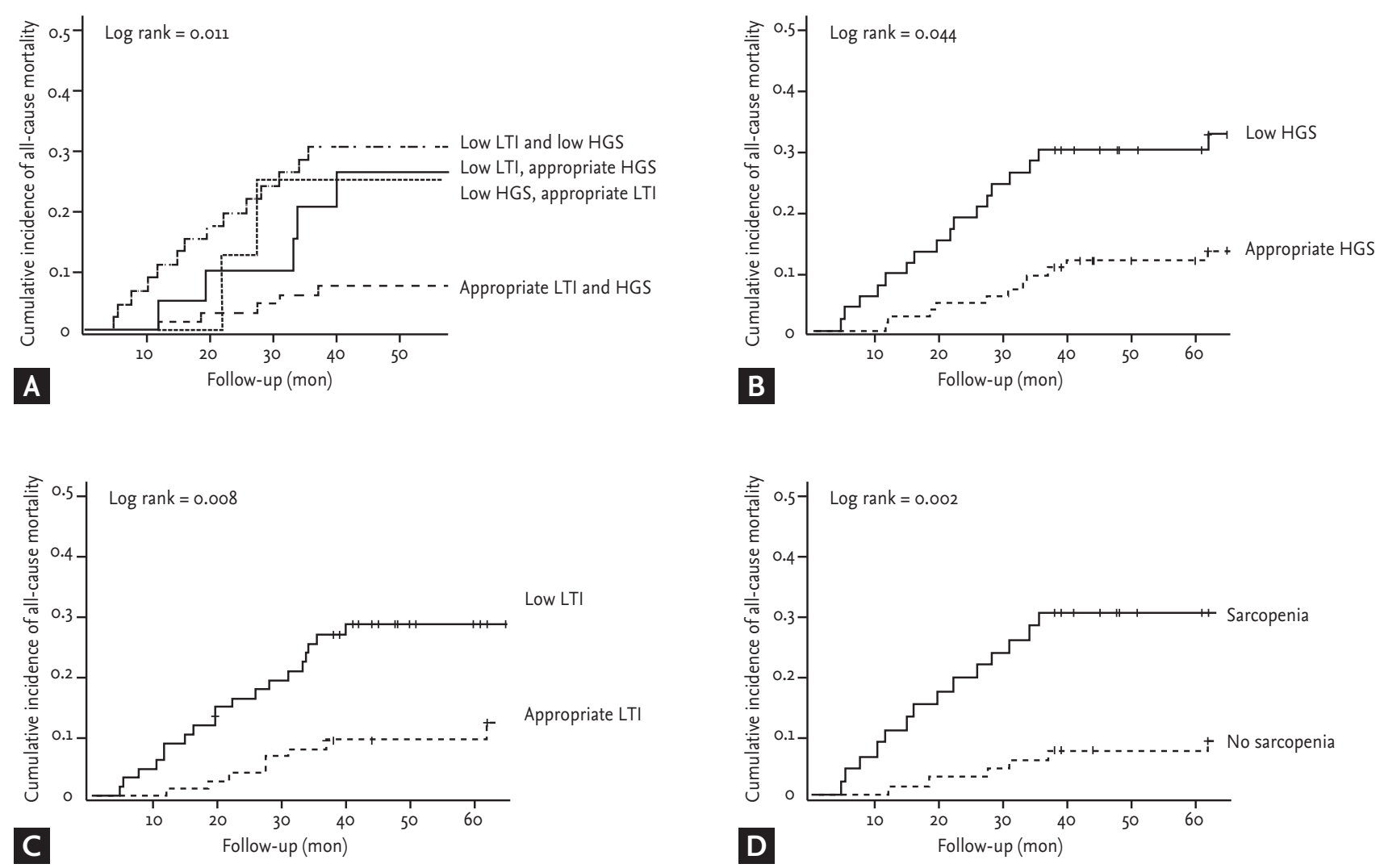

Figure 1. Kaplan-Meier survival probabilities for all-cause mortality based on the lean tissue index (LTI) and the handgrip strength (HGS). (A) Appropriate muscle mass with muscle strength had a significantly lower risk for mortality. Whereas, patients with (B) low muscle strength or (C) low muscle mass or (D) sarcopenia were at higher risk of death from all causes $(p<0.05$ by the log-rank test).

Table 2. Baseline nutritional status of patients according to survival status

\begin{tabular}{|c|c|c|c|}
\hline Variable & Deceased $(n=28,19.7 \%)$ & Survived $(\mathrm{n}=114,80.3 \%)$ & $p$ value \\
\hline Body mass index, $\mathrm{kg} / \mathrm{m}^{2}$ & $22.1 \pm 2.9$ & $22.5 \pm 3.4$ & 0.557 \\
\hline Triceps muscle thickness, mm & $10.1 \pm 3.8$ & $11.1 \pm 3.6$ & 0.343 \\
\hline Subjective Global Assessment, score & $5 \cdot 3 \pm 0.8$ & $6.0 \pm 1.0$ & 0.002 \\
\hline Handgrip strength, kg & $20.2 \pm 7.5$ & $25.2 \pm 8.6$ & 0.007 \\
\hline LTI, $\mathrm{kg} / \mathrm{m}^{2}$ & $11.3 \pm 2.4$ & $13.4 \pm 2.8$ & 0.001 \\
\hline Fat tissue index, $\mathrm{kg} / \mathrm{m}^{2}$ & $10.4 \pm 3.6$ & $8.8 \pm 4 \cdot 3$ & 0.092 \\
\hline Albumin, g/dL & $3.7 \pm 0.5$ & $3.8 \pm 0.3$ & 0.134 \\
\hline Prealbumin, mg/dL & $21.7 \pm 8.0$ & $27.7 \pm 8.9$ & 0.004 \\
\hline Low LTI & $19(67.9)$ & $48(42.1)$ & 0.014 \\
\hline Low HGS & $17(60.7)$ & $37(32.5)$ & 0.006 \\
\hline LTI and HGS categories (4 groups) & & & 0.016 \\
\hline Low LTI and Low HGS & $14(50.0)$ & $33(28.1)$ & \\
\hline Low HGS and appropriate LTI & $3(10.7)$ & $5(4 \cdot 4)$ & \\
\hline Low LTI and appropriate HGS & $5(17.9)$ & $15(13.2)$ & \\
\hline Appropriate LTI and HGS & $6(21.4)$ & $62(54 \cdot 4)$ & \\
\hline
\end{tabular}

Values are presented as mean $\pm \mathrm{SD}$ or number (\%).

LTI, lean tissue index; HGS, handgrip strength. 
Table 3. Correlation coefficient $(r)$ between various nutritional parameters and biochemical markers

\begin{tabular}{lccccccccc}
\hline Variable & LTI & FTI & HGS & BMI & Triceps & SGA & Albumin & Prealbumin & hs-CRP \\
\hline LTI & & $-0.481^{\mathrm{a}}$ & $0.544^{\mathrm{a}}$ & $0.15^{\mathrm{a}}$ & -0.106 & $0.243^{\mathrm{a}}$ & 0.041 & 0.014 & -0.146 \\
FTI & $-0.481^{\mathrm{a}}$ & & $-0.411^{\mathrm{a}}$ & $0.645^{\mathrm{a}}$ & $0.421^{\mathrm{a}}$ & -0.080 & 0.009 & 0.035 & 0.137 \\
HGS & $0.544^{\mathrm{a}}$ & $-0.411^{\mathrm{a}}$ & & 0.022 & -0.101 & $0.347^{\mathrm{a}}$ & 0.036 & 0.172 & $-0.241^{\mathrm{a}}$ \\
BMI & $0.18^{2}$ & $0.645^{\mathrm{a}}$ & 0.022 & & $0.489^{\mathrm{a}}$ & 0.158 & 0.184 & 0.113 & 0.103 \\
Triceps & -0.106 & $0.489^{\mathrm{a}}$ & -0.101 & $0.489^{\mathrm{a}}$ & & $0.281^{\mathrm{a}}$ & 0.155 & $0.384^{\mathrm{a}}$ & 0.060 \\
SGA & $0.243^{\mathrm{a}}$ & $0.15^{2}$ & $0.347^{\mathrm{a}}$ & $0.15^{2}$ & $0.281^{\mathrm{a}}$ & & 0.106 & $0.396^{\mathrm{a}}$ & -0.201 \\
Albumin & 0.041 & 0.184 & 0.036 & 0.041 & 0.155 & 0.106 & & $0.314^{\mathrm{a}}$ & -0.157 \\
Prealbumin & 0.014 & 0.113 & 0.172 & 0.014 & $0.384^{\mathrm{a}}$ & $0.396^{\mathrm{a}}$ & $0.314^{\mathrm{a}}$ & & -0.093 \\
hs-CRP & -0.146 & 0.103 & $-0.241^{\mathrm{a}}$ & 0.103 & 0.060 & -0.201 & -0.157 & -0.093 & \\
\hline
\end{tabular}

LTI, lean tissue index; FTI, fat tissue index; HGS, handgrip strength; BMI, body mass index; SGA, Subjective Global Assessment; hs-CRP, high-sensitivity C-reactive protein.

${ }^{a} p<0.05$.

${ }^{\mathrm{b}}$ Log-transformed.

Table 4. Multivariate Cox regression analyses for all-cause mortality and cardiovascular events

\begin{tabular}{|c|c|c|c|c|c|c|}
\hline \multirow{2}{*}{ Variable } & \multicolumn{3}{|c|}{ All-cause mortality } & \multicolumn{3}{|c|}{ Cardiovascular events } \\
\hline & Hazard ratio & $95 \% \mathrm{CI}$ & $p$ value & Hazard ratio & $95 \%$ CI & $p$ value \\
\hline Age & 1.06 & $1.00-1.02$ & 0.003 & 1.10 & $1.04-1.15$ & $<0.001$ \\
\hline Sex & 0.46 & $0.17-2.22$ & 0.457 & 0.25 & $0.05^{-1.21}$ & 0.084 \\
\hline Diabetes & 1.95 & $0.63-10.5$ & 0.437 & 1.08 & $0.15-7.90$ & 0.943 \\
\hline Coronary artery disease & $3 \cdot 57$ & $1.40-9.11$ & 0.008 & 7.07 & $2.11-23.74$ & 0.002 \\
\hline Cerebrovascular disease & 0.72 & $0.67-1.96$ & 0.518 & 1.44 & $0.51-4.12$ & 0.493 \\
\hline $\mathrm{BMI}, \mathrm{kg} / \mathrm{m}^{2}$ & 1.01 & $0.85-1.21$ & 0.907 & 1.20 & $1.00-1.44$ & 0.053 \\
\hline hs-CRP ${ }^{\mathrm{a}}$ & 2.53 & $1.73-3.68$ & 0.001 & 1.41 & $0.98-2.03$ & 0.640 \\
\hline SGA (B vs. A) & 6.71 & $2.13-21.08$ & 0.001 & 0.87 & $0.34-3.18$ & 0.827 \\
\hline Albumin & 0.39 & $0.10-1.57$ & 0.185 & 0.59 & $0.12-2.89$ & 0.512 \\
\hline Low lean tissue index & 2.77 & $1.10-6.97$ & 0.030 & 3.01 & $1.09-8.29$ & 0.033 \\
\hline Low handgrip strength & 5.65 & $1.99-16.04$ & 0.001 & 4.09 & $1.26-13.29$ & 0.019 \\
\hline Sarcopenia & 6.99 & $1.84-26.58$ & 0.004 & 4.33 & $1.51-12.43$ & 0.006 \\
\hline
\end{tabular}

Multivariate Cox regression analysis was performed after adjusting for age, gender, BMI, Kt/V, albumin, diabetes, dialysis vintage, hs-CRP and previous history of coronary artery disease and cerebrovascular disease.

CI, confidence interval; BMI, body mass index; hs-CRP, high-sensitivity C-reactive protein; SGA, Subjective Global Assessment. ${ }^{a}$ Log-transformed.

al probabilities for all-cause mortality based on the LTI and the HGS. Patients with low muscle mass or low muscle strength were at higher risk of death from all causes $(p$ $<0.05$ by the log-rank test). Clinical indicators associated with mortality are shown in Table 4. Old age, a low SGA, $\mathrm{CAD}$, and a high hs-CRP level were significantly associated with increased all-cause mortality risk. None of BMI, the FTI, or triceps muscle thickness affected survival. Both a low HGS (HR, 5.65; 95\% CI, 1.99 to 16.04; p $=0.001)$ and a low LTI (HR, 2.77; 95\% CI, 1.10 to 6.97; $p=$
0.030) were significantly associated with a higher risk of death. Sarcopenia was a strong predictor of cardiovascular events (HR, 4.33; 95\% CI, 1.51 to $12.43 ; p=0.006$ ) and mortality (HR, 6.99; 95\% CI, 1.84 to $26.58 ; p=0.004$ ).

\section{DISCUSSION}

Our principal findings in this study were that sarcopenia was prevalent and important predictor of all-cause 
mortality and the development of cardiovascular events in patients undergoing HD. None of BMI, the triceps skin-fold thickness, or the FTI (reflecting body fat composition) was associated with mortality. The SGA, CAD, and hs-CRP levels were significantly associated with mortality, even after adjustment for age, gender, diabetes status, and BMI.

Sarcopenia was originally defined as age-related skeletal muscle loss with associated muscular dysfunction. Today, however, the term is used to describe loss of muscle mass and function upon aging, development of chronic disease, physical inactivity, and inappropriate nutrition. Recently, the effect of sarcopenia on longterm clinical outcomes has become clearer; sarcopenia has become recognized as an important prognostic marker in various populations. In HD patients, sarcopenia strongly affects mortality. We found that approximately one-third (33.1\%) of current HD patients had sarcopenia; low muscle mass and low muscle strength increased the all-cause mortality risks 2.8- and 5.6-fold, respectively.

In patients with renal dysfunction, increased levels of uremic toxins and chronic inflammation may accelerate normal physiological muscle wasting and reduce muscle force [17]. Indeed, selective muscular structural changes, and significant muscle wasting, have been documented in patients with uremic conditions; the phenomenon has been termed "uremic sarcopenia" [18,19]. Deterioration of renal function is associated with many complications including fluid and metabolic abnormalities, and electrolyte and hormone imbalances. The cumulative effects of CKD and associated complications in terms of skeletal muscle wasting are usually amplified when progressive renal dysfunction is in play $[17,19,20]$.

Although the pathophysiological mechanisms have not been clear, malnutrition, chronic inflammation, and various changes in humoral factors in uremia may cause sarcopenia and resultant cardiovascular events. Low-grade systemic inflammation is very common in even early-stage CKD patients, contributing to the higher mortality rate of HD patients compared with that in the general population [21]. Increased chronic inflammation enhances muscle wasting $[22,23]$. Tumor necrosis factor- $\alpha$ (TNF- $\alpha$ ) increases muscle wasting by activating the nuclear factor- $\kappa \beta$ pathway; TNF- $\alpha$ attenuates insulin-stimulated protein synthesis [24]. Similarly, the infusion of cytokines including TNF- $\alpha$ and interleukin-6 increases muscle protein degradation mediated via the NF- $\kappa \beta$ pathway $[25,26]$. Metabolic acidosis may also explain the link between sarcopenia and increased mortality from CKD. Such acidosis is very common in patients with advanced renal failure; most prior observational studies found significant associations between metabolic acidosis and high-level mortality in patients with CKD in ESRD [27]. Metabolic acidosis promotes muscle protein wasting and degradation. One recent study found that sodium bicarbonate supplementation of patients with predialysis CKD slowed the decline in renal function, increased dietary protein intake, and reduced the level of normalized protein nitrogen. Thus, protein breakdown was reduced, improving lean body mass as measured by the mid-arm muscle circumference [28-30]. Reduction in protein degradation with preservation of muscle mass may explain why mortality is reduced after the correction of metabolic acidosis in dialysis patients [30]. Low vitamin D levels may also explain the observed association. Vitamin D status is positively associated with muscle strength [31], and physical performance [32], and inversely associated with the risk of falling [33]. We found that sarcopenic patients had significantly lower vitamin D levels than those without sarcopenia. This suggests that vitamin D deficiency may play a role in CKD myopathy; early clinical reports indeed recognized the potential association between vitamin D status and muscle strength in CKD patients. Also, sarcopenic patients commonly experience functional impairment and physical disability which causes a reduction in muscle contraction-induced factors such as myokines. As these myokines have an anti-inflammatory effect, the relative paucity of myokines in sarcopenia may increase the risk of CVD [34,35].

Of the two components of sarcopenia, muscle power has recently been recognized as being more important than muscle mass. Isoyama et al. [14] explored the influences of muscle mass and strength on the mortality of dialysis patients, and found that reduced muscle strength was more strongly associated with aging, PEW, physical inactivity, inflammation, and mortality than was low muscle mass. The cited authors emphasized that assessment of muscle functionality was critical in sarcopenia patients. In contrast, we found that both a low HGS and a low LTI affected mortality. These discrepancies may 
be explained by differences in the studied patients. The cited study included only patients starting on dialysis, but we included both such patients and those on longterm dialysis. Indeed, the median dialysis duration was 4 years in the present study. As the duration of dialysis increases, muscle mass may fall, associated with more severe disability and increased mortality.

Our study had several limitations. First, when we assessed sarcopenia, we did not measure physical performance. Many patients could not move at all without assistance from caregivers. We thus did not explore physical performance in detail. Second, we enrolled a relatively small number of patients, increasing the CIs around our predictions.

In conclusion, we found that both low LTI and low HGS, but especially the combination of both are associated with increased risk for all-cause mortality and cardiovascular events in patients undergoing HD. These results suggest that noninvasive assessment of LTI and HGS may provide additional prognostic information in HD patients.

\section{KEY MESSAGE}

1. Sarcopenia is prevalent in patients undergoing hemodialysis, and could be an important predictor of all-cause mortality and the development of cardiovascular events

2. None of body mass index, the triceps skin-fold thickness, or the fat tissue index was associated with mortality.

3. Assessment of muscle strength and muscle mass may provide additional prognostic information to survival in patients with end-stage renal disease.

\section{Conflict of interest}

No potential conflict of interest relevant to this article was reported.

\section{Acknowledgments}

This work was supported by a grant from Hallym University Medical Center Research Fund.

\section{REFERENCES}

1. Stenvinkel P, Heimburger O, Paultre F, et al. Strong association between malnutrition, inflammation, and atherosclerosis in chronic renal failure. Kidney Int 1999;55:18991911.

2. Metter EJ, Conwit R, Tobin J, Fozard JL. Age-associated loss of power and strength in the upper extremities in women and men. J Gerontol A Biol Sci Med Sci 1997;52:B267-B276.

3. Muscaritoli M, Anker SD, Argiles J, et al. Consensus definition of sarcopenia, cachexia and pre-cachexia: joint document elaborated by Special Interest Groups (SIG) "cachexia-anorexia in chronic wasting diseases" and "nutrition in geriatrics". Clin Nutr 2010;29:154-159.

4. Kalantar-Zadeh K, Abbott KC, Salahudeen AK, Kilpatrick RD, Horwich TB. Survival advantages of obesity in dialysis patients. Am J Clin Nutr 2005;81:543-554.

5. Lowrie EG, Li Z, Ofsthun N, Lazarus JM. Body size, dialysis dose and death risk relationships among hemodialysis patients. Kidney Int 2002;62:1891-1897.

6. Leavey SF, McCullough K, Hecking E, Goodkin D, Port FK, Young EW. Body mass index and mortality in 'healthier' as compared with 'sicker' haemodialysis patients: results from the Dialysis Outcomes and Practice Patterns Study (DOPPS). Nephrol Dial Transplant 2001;16:23862394.

7. Wolfe RA, Ashby VB, Daugirdas JT, Agodoa LY, Jones CA, Port FK. Body size, dose of hemodialysis, and mortality. Am J Kidney Dis 2000;35:80-88.

8. Kopple JD, Zhu X, Lew NL, Lowrie EG. Body weight-forheight relationships predict mortality in maintenance hemodialysis patients. Kidney Int 1999;56:1136-1148.

9. Sarkar SR, Kuhlmann MK, Kotanko P, et al. Metabolic consequences of body size and body composition in hemodialysis patients. Kidney Int 2006;70:1832-1839.

10. Carrero JJ, Chmielewski M, Axelsson J, et al. Muscle atrophy, inflammation and clinical outcome in incident and prevalent dialysis patients. Clin Nutr 2008;27:557-564.

11. Heimburger O, Qureshi AR, Blaner WS, Berglund L, Stenvinkel P. Hand-grip muscle strength, lean body mass, and plasma proteins as markers of nutritional status in patients with chronic renal failure close to start of dialysis therapy. Am J Kidney Dis 2000;36:1213-1225.

12. Roshanravan B, Robinson-Cohen C, Patel KV, et al. Association between physical performance and all-cause mor- 
tality in CKD. J Am Soc Nephrol 2013;24:822-830.

13. Kim JK, Choi SR, Choi MJ, et al. Prevalence of and factors associated with sarcopenia in elderly patients with endstage renal disease. Clin Nutr 2014;33:64-68.

14. Isoyama N, Qureshi AR, Avesani CM, et al. Comparative associations of muscle mass and muscle strength with mortality in dialysis patients. Clin J Am Soc Nephrol 2014;9:1720-1728.

15. Painter P, Roshanravan B. The association of physical activity and physical function with clinical outcomes in adults with chronic kidney disease. Curr Opin Nephrol Hypertens 2013;22:615-623.

16. Steiber AL, Kalantar-Zadeh K, Secker D, McCarthy M, Sehgal A, McCann L. Subjective Global Assessment in chronic kidney disease: a review. J Ren Nutr 2004;14:191200.

17. Carrero JJ, Stenvinkel P, Cuppari L, et al. Etiology of the protein-energy wasting syndrome in chronic kidney disease: a consensus statement from the International Society of Renal Nutrition and Metabolism (ISRNM). J Ren Nutr 2013;23:77-90.

18. Fahal IH, Bell GM, Bone JM, Edwards RH. Physiological abnormalities of skeletal muscle in dialysis patients. Nephrol Dial Transplant 1997;12:119-127.

19. Fahal IH. Uraemic sarcopenia: aetiology and implications. Nephrol Dial Transplant 2014;29:1655-1665.

20. Fouque D, Kalantar-Zadeh K, Kopple J, et al. A proposed nomenclature and diagnostic criteria for protein-energy wasting in acute and chronic kidney disease. Kidney Int 2008;73:391-398.

21. Stenvinkel $\mathrm{P}$, Alvestrand A. Inflammation in end-stage renal disease: sources, consequences, and therapy. Semin Dial 2002;15:329-337.

22. Kalantar-Zadeh K, Kopple JD. Relative contributions of nutrition and inflammation to clinical outcome in dialysis patients. Am J Kidney Dis 2001;38:1343-1350.

23. Zhang L, Wang XH, Wang H, Du J, Mitch WE. Satellite cell dysfunction and impaired IGF-1 signaling cause CKD-induced muscle atrophy. J Am Soc Nephrol 2010;21:419-427.

24. Langen RC, Schols AM, Kelders MC, Wouters EF, Janssen-Heininger YM. Inflammatory cytokines inhibit myogenic differentiation through activation of nuclear factor-kappaB. FASEB J 2001;15:1169-1180.

25. Mourkioti F, Rosenthal N. NF-kappaB signaling in skeletal muscle: prospects for intervention in muscle diseases. J Mol Med (Berl) 2008;86:747-759.

26. Delano MJ, Moldawer LL. The origins of cachexia in acute and chronic inflammatory diseases. Nutr Clin Pract 2006;21:68-81.

27. Kraut JA, Kurtz I. Metabolic acidosis of CKD: diagnosis, clinical characteristics, and treatment. Am J Kidney Dis 2005;45:978-993.

28. Verove C, Maisonneuve N, El Azouzi A, Boldron A, Azar R. Effect of the correction of metabolic acidosis on nutritional status in elderly patients with chronic renal failure. J Ren Nutr 2002;12:224-228.

29. Caso G, Garlick PJ. Control of muscle protein kinetics by acid-base balance. Curr Opin Clin Nutr Metab Care 2005;8:73-76.

30. Stein A, Moorhouse J, Iles-Smith H, et al. Role of an improvement in acid-base status and nutrition in CAPD patients. Kidney Int 1997;52:1089-1095.

31. Visser M, Deeg DJ, Lips P; Longitudinal Aging Study Amsterdam. Low vitamin D and high parathyroid hormone levels as determinants of loss of muscle strength and muscle mass (sarcopenia): the Longitudinal Aging Study Amsterdam. J Clin Endocrinol Metab 2003;88:5766-5772.

32. Bischoff-Ferrari HA, Dietrich T, Orav EJ, et al. Higher 25-hydroxyvitamin D concentrations are associated with better lower-extremity function in both active and inactive persons aged $>$ or $=60 \mathrm{y}$. Am J Clin Nutr 2004;80:752758.

33. Snijder MB, van Schoor NM, Pluijm SM, van Dam RM, Visser M, Lips P. Vitamin D status in relation to one-year risk of recurrent falling in older men and women. J Clin Endocrinol Metab 2006;91:2980-2985.

34. Pedersen BK, Steensberg A, Fischer C, et al. Searching for the exercise factor: is IL-6 a candidate? J Muscle Res Cell Motil 2003;24:113-119.

35. Pedersen BK. Muscle-to-fat interaction: a two-way street? J Physiol 2010;588(Pt 1):21. 